Historic, Archive Document

Do not assume content reflects current scientific knowledge, policies, or practices. 

6 SECTIONAN TO POMOLOGY WHOLESALE PRICE RIST

\section{SPRING 1916}

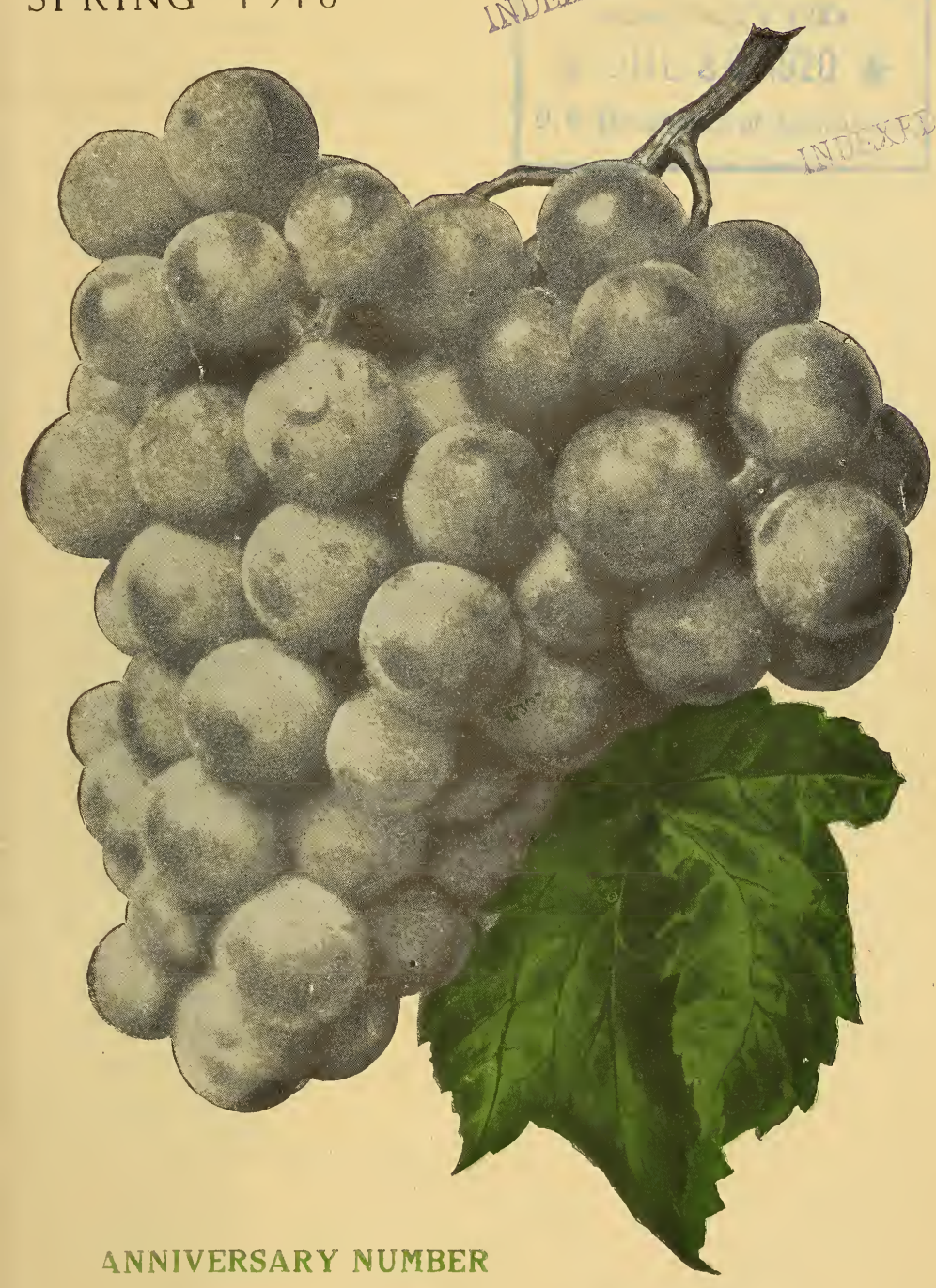

T. S. HUBBARD COMPANY

GRAPE VINE SPECIALISTS

FREDONIA

NEW YORK 


\section{FIFTIETH ANNIVERSARY}

This price list is the one-hundredth, semi-annual issue and completes fifty years in business.

In the fall of 1866 , the late T. S. Hubbard started growing Grape Vines and the plantings have increased until now we grow over two hundred acres annually.

It is our aim to furnish the best rooted vines that can be produced and graded to a uniform standard. The many thousands of vines growing in every grape section testify to the high quality and productiveness of our roots.

We thank our many customers for their patronage, which we appreciate and are pleased to say we still receive orders from several nurseries who were among the first to trade with us.

We have the largest stock in America and with our modern, concrete storage-cellar and packing-house can fill all orders very promptly:

We solicit an order during our Anniversary Year from those who have not dealt with us in recent years. 


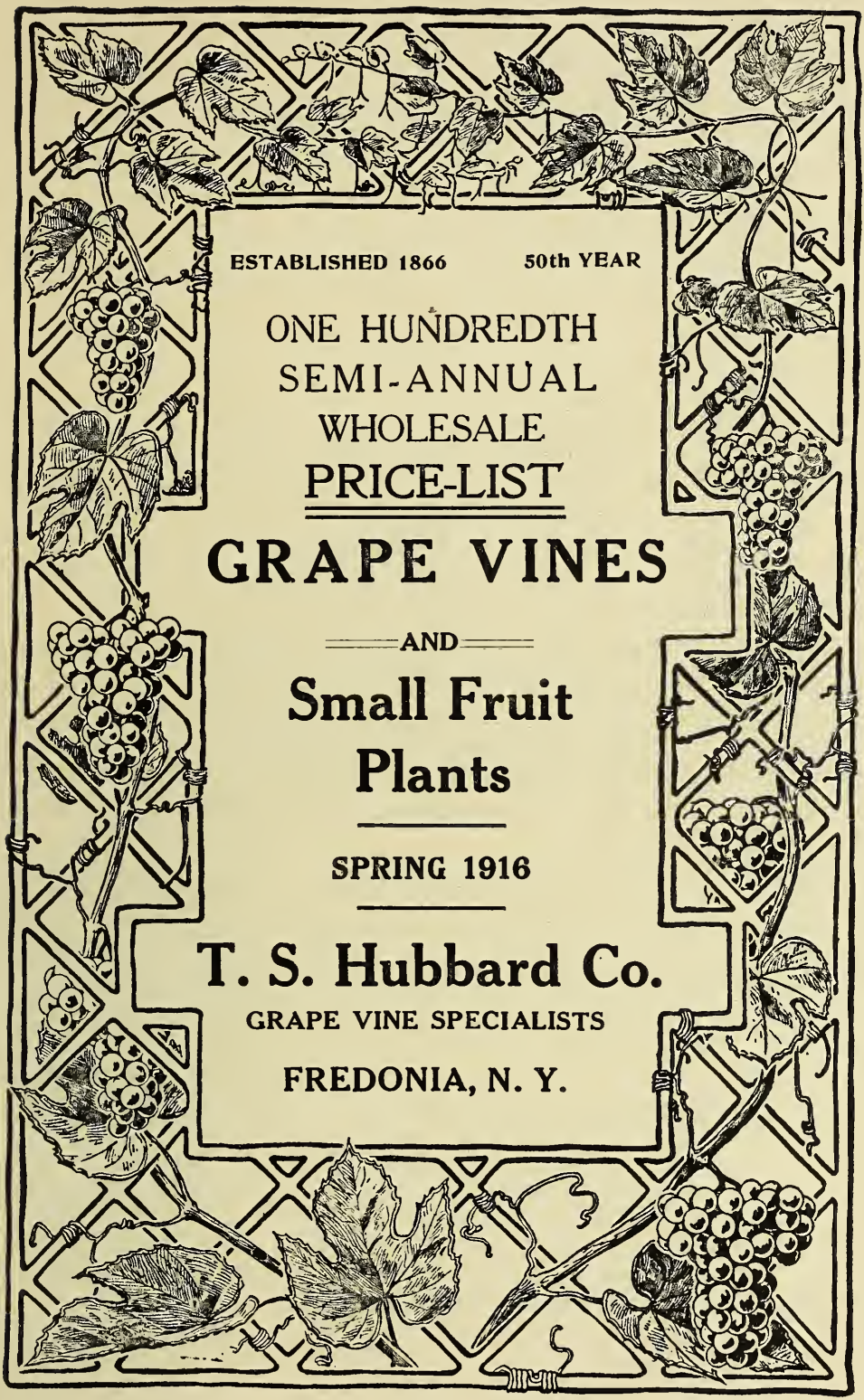




\section{Our specialte}

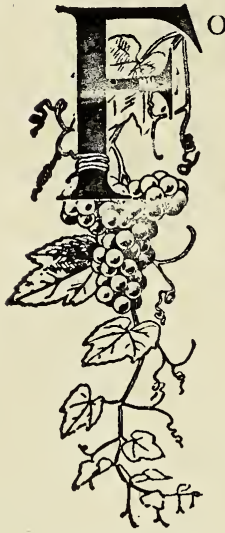

OR FIF'TY YEARS WE HAVE MADE THE growing of high grade GRAPE VINES a specialty. Our annual plantings average over two hundred acres, embracing many millions of vines, closely planted in nursery rows. We aim to keep in stock everything for the Planter or Dealer, all graded to our standard, known as the Fredonia Standard, which we originated many years ago, and now well known to the trade wherever grape vines are planted. Through our immense business and wide acquaintance we have established a national reputation for accuracy in varieties, grading to the highest standard, and promptness in filling orders, of which we are proud, and which it will always be our aim to deserve.

LOCATION-Three miles south of Dunkirk, forty miles west of Buffalo, on the L. S. \& M. S. R. R.; Erie R. R.; N. Y. Chi. \& St. L. (Nickel Plate) R. R.; Penn'a R. R.; and D. A. V. \& P. R. R. Electric cars connecting with all day and evening trains at Dunkirk go directly past our office and cellars, at 29 Central Avenue, every half hour during the day and evening. for boxes.

PACKING done in the most thorough manner. No charge

TIME OF SHIPMENT-October 1 to June 1. Our stock being stored in cellars, can be safely sent by mail or express during winter, or by freight, with special care in packing.

WE NEVER SUBSTITU'TE, but we reserve the right to fill orders for one-year vines with older vines of the same size, unless instructed to the contrary, and for two-year vines with extra large one-year of the same size as the two-year.

SAMPLES will be mailed to those wishing to purchase in quantity, at 100 rates with five cents added for postage on three one-year vines, and ten cents on three two-year vines.

ACCEPTANCE OF ORDERS-It is mutually understood and agreed between this company and the purchaser that all orders accepted by the company are subject to the following conditions, viz:

1. The company guarantees all stock to be in good healthy condition at the time and point of shipment.

2. It shall not be held liable for loss of or damage to stock in transit. 
3. It warrants all stock true to name and of quality represented, with the express understanding and agreement with the purchaser that, should any prove not true to name it will refund the money paid, or will replace with other stock, but shall not be liable to damages other than herein named.

4. The purchaser waives all claims and damages against this company resulting from failure to fill orders where delivery is prevented by the loss of the company's stock by fire or the elements before shipment.

5. All orders shall be filled subject to the above conditions.

\section{TERMS}

CASH with the order, or satisfactory references from unknown correspondents.

ALL BILLS must be paid by June 1st, unless otherwise agreed.

FOR ALL BILLS not paid by June 1st, we will draw at sight without notification.

INTEREST charged on all bills overdue. Note payable at Bank for 30 or 60 days will be accepted on satisfactory references.

IF TO BE SHIPPED C. O. D., one-fourth of the bill or satisfactory references required with order from unknown correspondents ; return charges on money to be paid by buyer.

AS THE SEASON advances we are likely to be short of some varieties and we reserve the right to advance the prices without notice, or decline to allow the discounts offered.

We advise our customers to order early.

\section{GRAPE VINES}

We can furnish one year medium vines (a grade smaller than number one) at $70 \%$ the price of one year number one.

Also can furnish three year vines, heavy, in nearly all leading kinds at half additional to price of two year number one.

Weight of vines packed for shipment:

1 -year No. 1 , about 140 to 180 pounds per 1,000 .

1 -year No. 2, abuut 100 to 150 pounds per 1,000 .

2 -year No. 1, about 280 to 350 pounds per 1,000 .

These are about the average weights for a single thousand, including box. Where a number of thousand are shipped in the same box, the weight will average from one-fifth to onethird less, according to quantity.

We will quote freight or express rates to any point on application. 


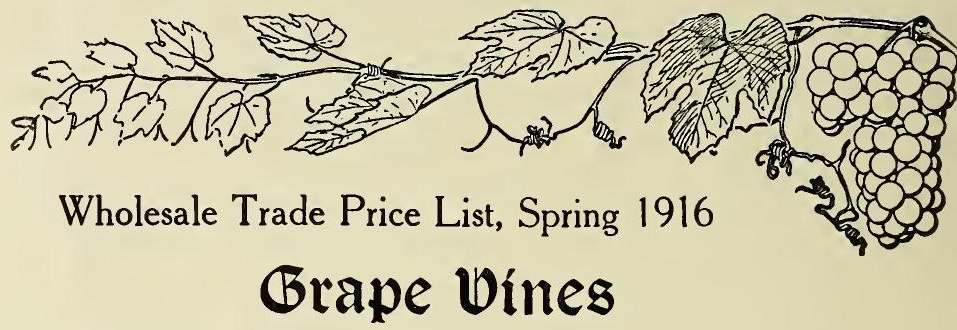

Box and packing free.

These prices are subject to change without notice.

\begin{tabular}{|c|c|c|c|c|}
\hline LEADING VARIETIES & \multicolumn{2}{|c|}{ One-year No, 1} & \multicolumn{2}{|c|}{ Two-year. No. 1} \\
\hline Agawam (Rog, No, 15) & S? 50 & & & \\
\hline $\begin{array}{l}\text { Agawam (Kog. No. 10) } \\
\text { Beta }\end{array}$ & $\begin{array}{r}\$ 250 \\
600\end{array}$ & $\begin{array}{r}\$ 2200 \\
5000\end{array}$ & $\begin{array}{r}\$ 400 \\
800\end{array}$ & $\begin{array}{r}\$ 3500 \\
7500\end{array}$ \\
\hline Brighton...... & 500 & 4000 & 700 & 6000 \\
\hline Campbell's Early....................... & 600 & 5000 & 900 & 7500 \\
\hline Catawba...................... & 250 & 2000 & 350 & 3000 \\
\hline Champion or Talman........... & 250 & 1800 & 350 & 2500 \\
\hline Concord................. & 200 & 1600 & 300 & 2500 \\
\hline 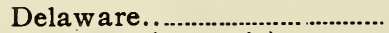 & 400 & 3500 & 600 & 5000 \\
\hline Diamond (Moore's).................... & 350 & 3000 & 500 & 4500 \\
\hline Duchess............... & 450 & 4000 & 650 & 6000 \\
\hline Early Ohio.... & 650 & 6000 & 1000 & 9000 \\
\hline Eaton & 900 & 8000 & 1300 & 12000 \\
\hline ................................. & 300 & 2500 & 400 & 3500 \\
\hline 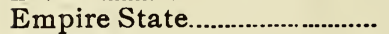 & 400 & 3500 & 600 & 5000 \\
\hline Green Mountain......................... & 1200 & 10000 & 1800 & 15000 \\
\hline 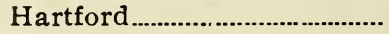 & 400 & 3500 & 600 & 5000 \\
\hline Iona........ & 350 & 3000 & 500 & 4500 \\
\hline Ives.......... & 300 & 2500 & 450 & 35 \\
\hline Jefferson & 1000 & 8000 & 1500 & 12000 \\
\hline (............................. & 500 & 4000 & 700 & 6000 \\
\hline Lindley (Rog. No. 9) ............. & 300 & 2500 & 450 & 3500 \\
\hline 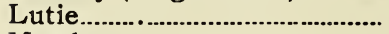 & 600 & 5000 & 800 & 7500 \\
\hline Martha.. & 400 & 3500 & 600 & 5000 \\
\hline Massasoit (Rog. No. 3) ...... & 400 & 3500 & 600 & 5000 \\
\hline Moore's Early.. & 350 & 3000 & 500 & 4500 \\
\hline 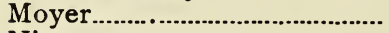 & 300 & 2500 & 450 & 4000 \\
\hline Niagara. & 350 & 2500 & 600 & 5000 \\
\hline ......................... & 600 & 5000 & 800 & 7500 \\
\hline Pocklington & 400 & 3500 & 600 & 5000 \\
\hline 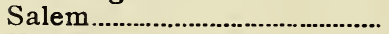 & 400 & & 600 & \\
\hline 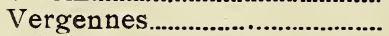 & 400 & 35 & 600 & 5000 \\
\hline .................... & 500 & 4000 & 700 & 6000 \\
\hline Woodruff Red......... & 600 & 50 & 800 & \\
\hline 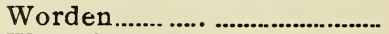 & 300 & 2500 & 400 & 35.00 \\
\hline 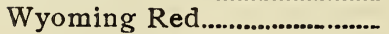 & 400 & 3500 & 600 & 5000 \\
\hline
\end{tabular}




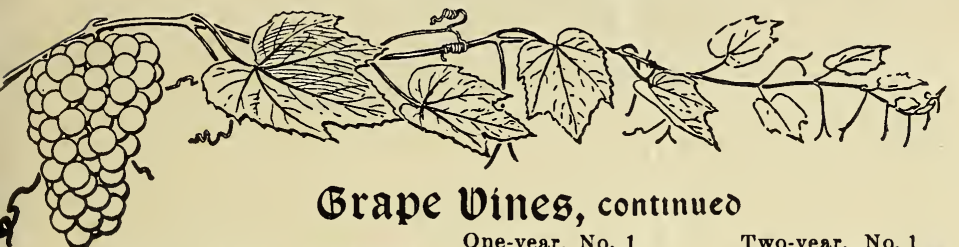

ADditional VARIETIES Per 100 , No. 1

Amber Queen

August Giant.

Bacchus

Barry (Rog. No. 43)

Berckman's

Brilliant

Clinton

Cottage

Cynthiana

Diana

Dracut Amber

Early Victor

Esther.

Etta

Eumelan

Gaertner (Rog. No. 14)

Geneva

Goethe (Rog. No. I)

Green's Early

Hayes.

Herbert (Rog. No. 44)

Herman Jaeger..

Isabella

Janesville

Jessica

Lucile

Mo. Reissling.

Perkins

Prentiss

Rommel

Telegraph

Triumph

Ulster Prolific. $\$ 600$

600

350

700

800

1000

250

300

500

500

$\$ 5000$

550 n

3000

6000

7500

9000

2000

2500

4500

3500

3000

3000

7500

3000

4000

7500

5000

6500

6500

3500

5000

550

1500

350

400

800

600

300

550

800

600

400

1200

600
Two-year, No. 1

Per 100 Per 1,000

$\$ 800 \$ 7500$

$900 \quad 8000$

$500 \quad 4500$

$1000 \quad 5000$

$1100 \quad 10000$

$1500 \quad 12500$

$350 \quad 3000$

$500 \quad 4000$

$700 \quad 6500$

$600 \quad 5000$

$500 \quad 4500$

$500 \quad 4500$

$1100 \quad 10000$

$500 \quad 4500$

$700 \quad 6000$

$1200 \quad 10000$

$800 \quad 7500$

$1000 \quad 9500$

$1000 \quad 9500$

$600 \quad 5000$

$800 \quad 7500$

2200

$500 \quad 4500$

$600 \quad 5000$

1200

$800 \quad 7500$

$500 \quad 4000$

$800 \quad 7500$

$1300 \quad 12000$

$800 \quad 7500$

$600 \quad 5000$

1800

$800 \quad 7500$

\section{DISCOUNTS.}

Discounts apply, to everything offered in this list.

$\$ 3$ to $\$ 10$ worth at 100 rates.

$\$ 10$ to 25 worth at 1,000 rates.

$\$ 25$ to 50 worth at 10 per cent off from 1,000 rates.

$\$ 50$ to 100 worth at 15 per cent off from 1,000 rates.

For over $\$ 100$ worth send list for us to price, stating grade and number of each variety wanted. 


\section{small Jruits}

In connection with our specialty, Grape Vines, we also grow a large stock of small fruit plants including all leading, well tested varieties.

\section{CURRANTS}

RE D VA RIETIES

\begin{tabular}{|c|c|c|c|c|}
\hline \multirow[b]{3}{*}{ horry } & \multicolumn{2}{|c|}{ One-year, No. 1} & \multicolumn{2}{|c|}{ Two-year, No. 1} \\
\hline & Per 100 & Per 1,000 & Per 100 & Per $1, C 00$ \\
\hline & $\$ 300$ & $\$ 2000$ & $\$ 400$ & $\$ 3000$ \\
\hline Fay's Prolific... & 300 & 2000 & 400 & 3000 \\
\hline 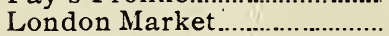 & 300 & 2000 & 400 & 3000 \\
\hline 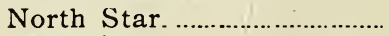 & 200 & 1600 & 300 & 2200 \\
\hline Perfection & 900 & 8000 & 1100 & 10000 \\
\hline President Wilder... & 300 & 2000 & 400 & 3000 \\
\hline Pomona..................... & 300 & 1800 & 400 & $25 \mathrm{co}$ \\
\hline Prince Albert & 300 & 1800 & 400 & 2500 \\
\hline Red Cross................................ & 300 & 2000 & 400 & 3000 \\
\hline 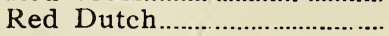 & 200 & 1500 & 300 & 2000 \\
\hline 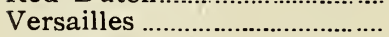 & 300 & 2000 & 400 & 3000 \\
\hline Victoria. & 200 & 1400 & 300 & 2000 \\
\hline W H I T E & V A R I E & T I E S & & \\
\hline White Dutch. & 300 & 2000 & 400 & 3000 \\
\hline White Grape & 300 & 2000 & 400 & 3000 \\
\hline White Imperial............................. & 300 & 2000 & 400 & 3000 \\
\hline B L A C K & V A R I E & T IES & & \\
\hline Black Champion.......... & 300 & 2000 & 400 & 3000 \\
\hline lack Naples............................. & 300 & 2000 & 400 & 3000 \\
\hline Boskoop Giant & 300 & 2500 & 400 & 3000 \\
\hline Lee's Prolific...................... & 300 & 2000 & 400 & 3500 \\
\hline
\end{tabular}

\section{GOOSEBERRIES}

RED VARIETIES

Houghton $250 \quad 2000$

600 3000

Industry.

Red Jacket (Josselyn) $400 \quad 3500$

500

4500 WHITE AND YELLOW VARIETIES

Chautauqua

Columbus.

Downing.

Pearl

Smith's Improved

Triumph
$\$ 900$

800

300

300

400

600
$\$ 7500$ 6000 2500 2500

3500

5000
$\$ 1200$ 1000 400 400 600 800
10000 9000 3500 3500 5000 7500 


\section{BLACKBERRIES}

\section{Root-cutting plants}

Our Blackberry plants have the same mass of fibrous roots as our grape vines and are superior in every way to those usually handled in the trade.

\begin{tabular}{|c|c|c|}
\hline Agawam & $\begin{array}{ll}\text { Per } & 100 \\
\$ 2 & 00\end{array}$ & $\begin{array}{r}\text { Per } 1,000 \\
\$ 1500\end{array}$ \\
\hline Ancient Briton ..... & 200 & 1500 \\
\hline Blowers' ......... & 200 & 1500 \\
\hline Eldorado ........... & 200 & 1500 \\
\hline Erie ....................... & 200 & 1500 \\
\hline Lucretia Dewberry........ & 150 & 1000 \\
\hline Mersereau.................. & 200 & 1500 \\
\hline Minnew aski............ & 200 & 1500 \\
\hline Rathbun ....................... & 200 & 1500 \\
\hline Snyder ......... & 150 & 1000 \\
\hline 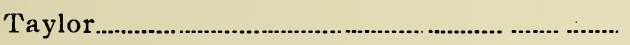 & 200 & 1500 \\
\hline Ward . ...................... & 200 & 1500 \\
\hline Wilson's Early .............. & 200 & 1500 \\
\hline
\end{tabular}

\section{RASPBERRIES}

Columbian

Cuthbert...

Early King.

Herbert.

Marlboro

120

1000

Miller

150

1200

St. Regis

200

Black Diamond

Cumberland

Kansas

Plum Farmer. 


\section{STRAWBERRIES}

Well rooted plants tied 25 in a bunch.

\begin{tabular}{|c|c|c|}
\hline andywin & $\begin{array}{l}\text { Per } 100 \\
\text { \$0 } 60\end{array}$ & $\begin{array}{r}\text { Per } 1000 \\
\$ 450\end{array}$ \\
\hline $\begin{array}{l}\text { andywin } \\
\text { bach }\end{array}$ & $\begin{array}{r}\$ 0 \\
60\end{array}$ & $\begin{array}{r}\$ 450 \\
400\end{array}$ \\
\hline$-1-1-1$ & 60 & 375 \\
\hline dy -..- & 60 & 400 \\
\hline Mary - & 60 & 375 \\
\hline and & 60 & 400 \\
\hline els Early & 60 & 400 \\
\hline N York & 60 & 400 \\
\hline Beauty_............ & 60 & 400 \\
\hline . & 60 & 4 \\
\hline - - - & 60 & 400 \\
\hline earing & 150 & \\
\hline iam Bel & 60 & 400 \\
\hline le Jim.. & 60 & \\
\hline
\end{tabular}

\section{SPECIAL NOTICE}

We have had a particularly favorable growing season and all our stock has made the strongest and best growth both in root and top ever offered. Dealers who require heavy plants for retail trade are requested to send us their want list for prices.

We call special attention to our large and fine stock in Currants, Gooseberry and Blackberry root-cutting plants.

We urge early orders to insure their being filled in full before our assortment is broken.

\section{COPY OF CERTIFICATE OF INSPECTION}

A Certificate of Inspection will be sent with every shipment and where State laws require it stock will be fumigated with hydrocyanic gas of the strength usually employed for that purpose.

\section{FOR HURRY ORDERS}

Long Distance Telephone in our Office connecting with all parts of the country.

In ordering by Telegraph use American Association of Nurserymen Telegraphic Code. 


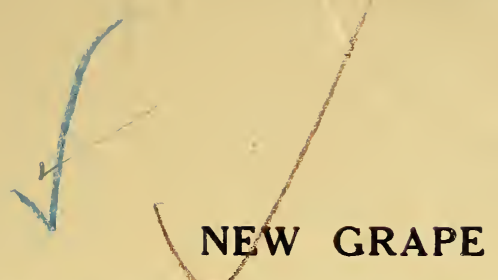

It is very fitting that in our Anniversary Year we announce the introduction of our new grape

\section{"HUBBARD"}

named in honor of the late T. S. Hubbard, founder of this Nursery and who did so much in the interest and advancement of the American Grape.

\section{DESCRIPTION.}

The Hubbard Grape originated in 1903.

Color, black. Clusters good size and shape. Berries large and uniform, averaging with wellgrown Concord. Adheres well to stem. Skin thin and tough. Does not crack easily. Seeds few and small and separate easily from pulp. Sweet, juicy, best quality, approaching Vinifera. Ripens about ten days before Concord.

We have investigated many new grapes in the past ten years and found no other grape with so many excellent points.

The Hubbard grape being of better quality, a better shipper, better size, healthy foliage, strong grower and earlier ripening makes it a more desirable grape to plant than either Concord or Niagara.

No vines offered, or orders booked, this spring. Further notice and full particulars will be made later. 


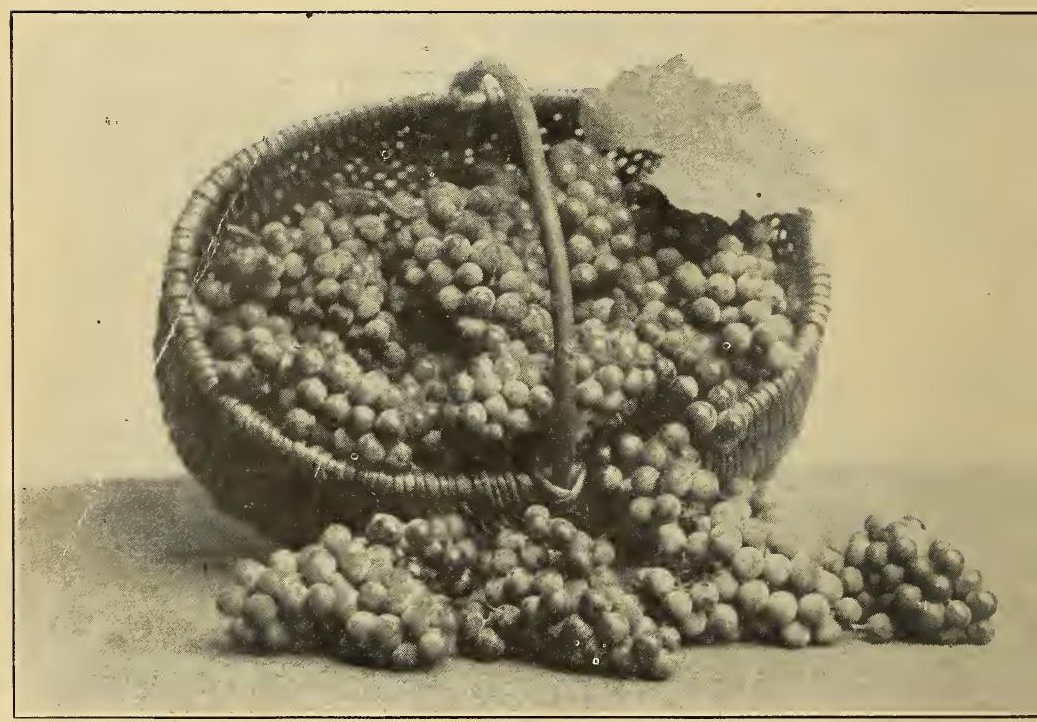

GRAPES GROWN FROM GOOD ROOTS 